\title{
Characterization of Fruit-Type Sucrose Synthase Gene Promoters Isolated from Tomato and Japanese Pear
}

\author{
Akio OHYAMA $^{1 *}$, Koji TANASE ${ }^{2}$, Keita SUWABE ${ }^{1,3}$, Miyuki KUNIHISA ${ }^{1,4}$, \\ Tsukasa NUNOME ${ }^{1}$ and Hiroyuki FUKUOKA ${ }^{1}$ \\ ${ }^{1}$ Molecular Genetics and Physiology Research Team, National Institute of Vegetable and Tea Science, \\ National Agriculture and Food Research Organization (NARO) (Tsu, Mie 514-2392, Japan) \\ ${ }^{2}$ Research Team for Breeding Technology, National Institute of Floricultural Science, NARO \\ (Tsukuba, Ibaraki 305-8519, Japan) \\ ${ }^{3}$ Faculty of Bioresources, Mie University (Tsu, Mie 514-8507, Japan)
}

\begin{abstract}
We isolated the 5' upstream promoter regions of the fruit-type sucrose synthase (SS) gene from tomato and Japanese pear by inverse PCR. The 5' region of the tomato SS gene (TOMSSF) contained an intron approximately $1.6 \mathrm{kbp}$ long in the 5 'untranslated region, whereas the equivalent 5 ' region in the Japanese pear SS gene (PypSUS1) had no intron. Each region was fused to the $\beta$-glucuronidase (GUS) gene and then each construct (TOMSSF 5'::GUS or PypSUS1 5'::GUS) was used to transform tomato. Histochemical analysis of GUS activity of all transformants showed high GUS activity in the fruit. Analysis of the staining pattern in the fruits of all transformants showed staining specific to vascular tissues and testae. The highest level of GUS activity and GUS mRNA was found in fruits at earlier stages of development in all transformants. However, the increase in the level was not observed in the ripening fruits of PypSUSI $5 ':: G U S$ lines, indicating that the expression patterns of PypSUS1 $5^{\prime}:: G U S$ tomato were different from those of PypSUS1 in Japanese pear. It may ascribe to the differences in genetic background between tomato and Japanese pear that relate to the mechanisms contributing to the sucrose synthesis during ripening.
\end{abstract}

Discipline: Biotechnology

Additional key words: promoter, sucrose synthase (EC 2.4.1.13)

\section{Introduction}

Sucrose synthase (SS) is an agriculturally important enzyme that catalyses the first mobilization reaction of transported sucrose in sink tissues, including crop seeds ${ }^{1,722}$, potato tubers ${ }^{4,14}$, and various fruits ${ }^{18,20}$. It is generally thought that SS participates in supplying fructose and uridine diphosphoglucose by cleaving sucrose to meet the metabolic demand of processes including starch synthesis, cell wall synthesis and energy generation ${ }^{17}$.

Multiple forms of SS exist in a range of different plants $^{7,17,18}$. The activity of fruit-type SS in tomato, known as the TOMSSF enzyme, is high in fruit at a young developmental stage and then decreases as the fruit matures ${ }^{9,13,20,23}$.
Therefore, production of the TOMSSF enzyme is believed to accompany the transient starch accumulation observed in young green fruit ${ }^{13,16,20}$. By comparison, production of the fruit-type SS in Japanese pear, known as the PypSUS1 enzyme, increases in fruit during the young developmental stage, declines as the fruit enlarges, and then increases again during the ripening process ${ }^{19}$. This unique expression profile of PypSUS1 implies that the increase in the enzyme level in ripening fruit is associated with sucrose resynthesis ${ }^{18,19}$.

Despite intensive expression analyses of the SS genes in fruits, including tomato and Japanese pear ${ }^{5,19-21}$, the promoters of these genes have not been well characterized. Fruit is a large sink organ to be harvested. Promoters driving genes in fruit are important tools for molecular improvement of fruit characteristics and molecular farming for production

\footnotetext{
Present address:

${ }^{4}$ Leaf and Root Vegetables Production Research Team, National Institute of Vegetable and Tea Science, NARO

(Tsukuba, Ibaraki 305-8666, Japan)

*Corresponding author: e-mail aohyama@affrc.go.jp

Received 28 November 2008 ; accepted 12 March 2009.
} 
of functional and/or valuable components in fruit. Here we report the isolation and characterization of the 5' upstream promoter region of the fruit-type SS gene in tomato and Japanese pear. The localization of SS activity was analyzed in tomato plants transformed with promoter-GUS fusion gene constructs.

\section{Materials and methods}

\section{Plant materials}

Leaves of Japanese pear (Pyrus serotina Rehder var. culta Rehder cv. Hosui) were kindly provided by Mie Prefecture Agricultural Research Institute (Matsusaka, Mie). Leaves of tomato (Solanum lycopersicum cv. Syugyoku) were harvested at a greenhouse of the National Institute of Vegetable and Tea Science.

\section{Isolation of $5^{\prime}$ upstream regions of the SS genes}

Genomic DNA was isolated from leaves of tomato and Japanese pear by the standard method ${ }^{8}$. The 5 ' upstream promoter regions of fruit-type SS genes were isolated from tomato and Japanese pear by inverse PCR (IPCR) ${ }^{3}$. For isolation of the 5' upstream region of the tomato $\mathrm{SS}$ gene, TOMSSF (DDBJ/GenBank/EMBL accession numbers AJ011535 and L19762), genomic DNA of tomato was digested by MunI and then self-ligated. The self-ligated DNA was digested by $A f l \mathrm{II}$ and then used as a template for IPCR. The 5' region of TOMSSF (TOMSSF 5') was PCR amplified by using the primers LeSUS485-505 (5'-CACTCAGGTAATTCGGGTTTG-3') and LeSUS6041 (5'-TAGTTGATTCAGCAGATGGG-3'). For isolation of the $5^{\prime}$ upstream region of the Japanese pear SS gene, PypSUS1 (accession number AB045710), genomic DNA of Japanese pear was digested by $C l a \mathrm{I}$ and then self-ligated. The self-ligated DNA was digested by $A f$ III and then used as a template for IPCR. The 5' region of PypSUS1 (PypSUS1 5') was PCR amplified by the primers PypSUS148-166 (5'-GACACTCTCTCCGACCACC-3') and PypSUS54-34 (5'- CTTCGTCTGTGTTCTGCAAGT -3'). A thermal cycle consisting of $20 \mathrm{~s}$ at $95^{\circ} \mathrm{C}, 30 \mathrm{~s}$ at $62^{\circ} \mathrm{C}$, and $5 \mathrm{~min}$ at $72^{\circ} \mathrm{C}$ was repeated 25 times. PCR products were cloned into the pGEM-T vector (Promega KK, Tokyo, Japan) and sequenced by an automated sequencer (model 373A; Applied Biosystems, Foster City, CA, USA). The cloned 5' upstream regions from TOMSSF (approximately $3.4 \mathrm{kbp}$ in length) and from PypSUS1 (approximately $1.8 \mathrm{kbp}$ in length) were designated as pTSPM1 and pPSP511-5, respectively.

\section{Estimation of transcription start sites of the promoters by 5 '-RACE analysis}

Transcription start sites of the TOMSSF 5' and PypSUS1 5 ' were estimated by 5 '-rapid amplification of cDNA ends
(5'-RACE) using the SMART RACE cDNA amplification kit (Clontech Laboratories, Inc., Palo Alto, CA, USA). RNA samples of young Japanese pear fruit (cv. Hosui) were kindly provided by Dr. S. Yamaki of Nagoya University. Tomato total RNA was isolated from Micro-Tom fruit at 35 to 45 days after anthesis (DAA) according to the method of Ohyama et al. ${ }^{11}$. Primers used for 5'-RACE analysis of TOMSSF and PypSUS1 mRNAs were TSPRACE+200a (5'-AATTGCATCGAACTCAGCCAAAAG-3') and PyPSSRACE+400a (5'-CGGTCAATTCCTCCACACTTAG3'), respectively. The PCR-amplified 5' region of the cDNA was cloned into the pT7 Blue vector (Novagen, Inc., Madison, WI, USA) and then sequenced.

\section{Construction of binary vectors}

The 5' upstream regions from TOMSSF in pTSPM1 and from PypSUS1 in pPSP511-5 were inserted into the upstream region of the GUS gene of pBI $101^{6}$ using standard molecular techniques. The resultant plasmids were designated as pTSSG-7 (TOMSSF 5 '::GUS, Fig. 1) and pPSSG-8 (PypSUS1 5'::GUS, Fig. 1), respectively.

\section{Rhizobium-mediated transformation of tomato}

Transformation of tomato (Solanum lycopersicum cv. Micro-Tom) was performed as described previously ${ }^{12}$. Rhizobium radiobactor (formerly Agrobacterium tumefaciens) LBA4404 harboring pTSSG-7 or pPSSG-8 was used as a donor. Regenerated transgenic tomatoes were grown in a greenhouse during the autumn and winter seasons and flowers of transformants were tagged at anthesis.

\section{Histochemical and enzymatic analyzes of GUS activity}

Histochemical and enzymatic analyses of transformed plants were performed as described by Ohta et al. ${ }^{10}$.

\section{Northern blot analysis of GUS expression}

Total RNA was extracted from transformed Micro-Tom fruit by the method of Ohyama et al. ${ }^{11}$. Samples of total RNA ( $5 \mu \mathrm{g} /$ lane) was denatured by treatment with formaldehyde and fractionated by agarose gel electrophoresis ${ }^{15}$. The RNA was transferred to a nylon membrane (Hybond $\mathrm{N}+$, GE Healthcare Bio-Sciences KK, Tokyo, Japan) and then probed with the GUS gene $(1.8 \mathrm{kbp})$ labeled with digoxigenin (DIG, Roche Diagnostics GmbH, Mannheim, Germany). Labeling of the GUS gene was performed by PCR amplification with the DIG DNA labeling mixture, the pBI221 plasmid template ${ }^{6}$, the GUS-F primer (5'ATGTTACGTCCTGTAGAAA-3'), and the GUS-R primer (5'-TCATTGTTTGCCTCCCTGCT-3') according to the manufacturer's protocol. Detection of hybridized DNA was performed by using the DIG Luminescent Detection Kit 


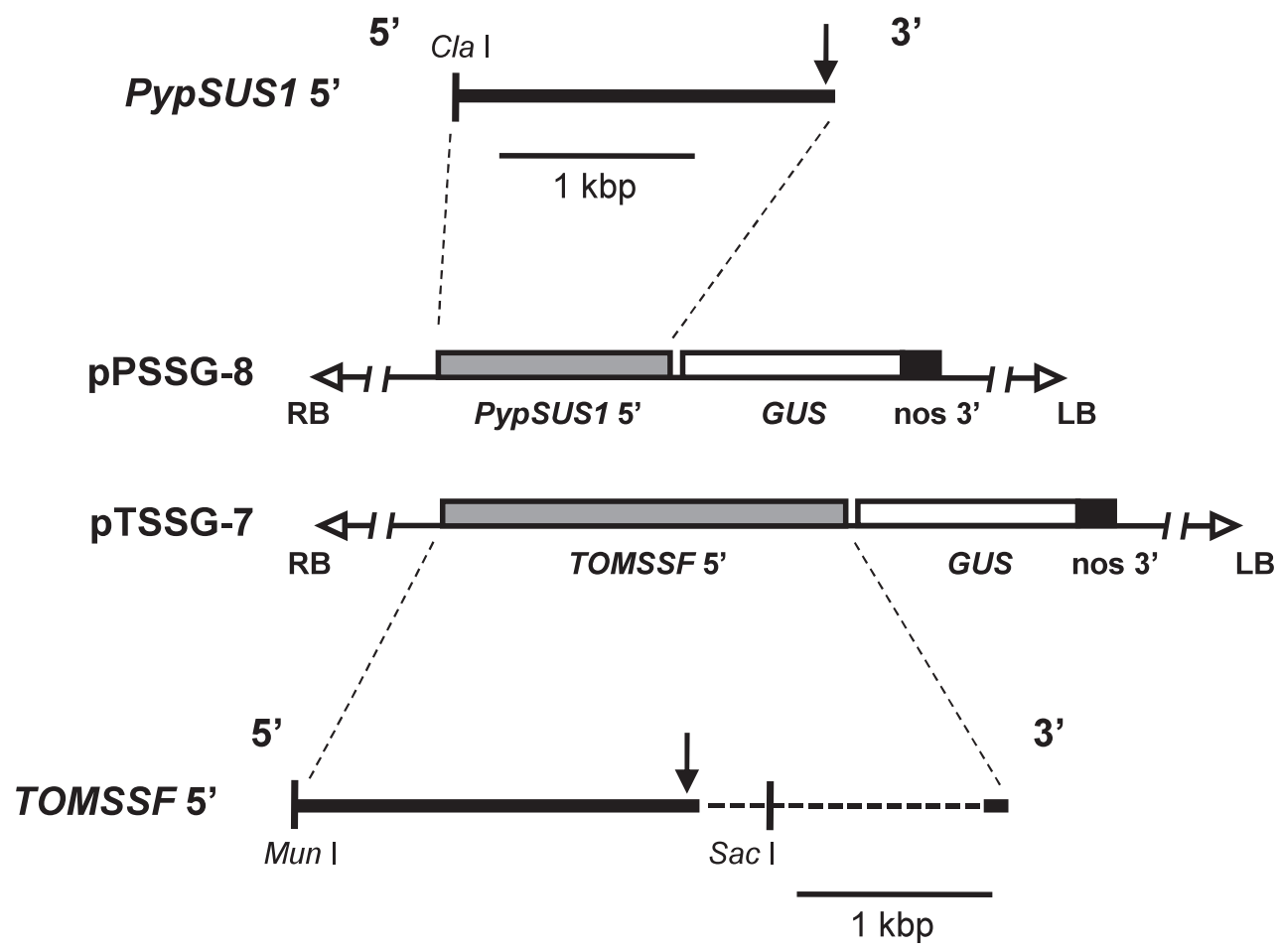

Fig. 1. Structure of the 5' upstream region of TOMSSF encoding tomato SS and PypSUS1 encoding Japanese pear SS, and construction of the binary vectors, pTSSG-7 and pPSSG-8

Arrows indicate the transcription start sites. The 5' regions were amplified by IPCR and then each region was inserted into the upstream of the GUS gene of pBI101 ${ }^{6}$. Resultant plasmids were designated as pTSSG-7 (TOMSSF 5'::GUS) and pPSSG8 (PypSUS1 5'::GUS). The construct pTSSG-7 contained approximately $3.4 \mathrm{kbp}$ of the TOMSSF 5', whereas pPSSG-8 contained approximately $1.8 \mathrm{kbp}$ of the PypSUS1 5'. LB and RB indicate the left and right borders of T-DNA, respectively. The dashed line indicates an intron approximately1.6 kbp in length at the 3' end of the TOMSSF 5'. The restriction sites for ClaI, MunI and SacI are shown.

(Roche Diagnostics GmbH).

\section{Results}

The 3' terminal sequences of the IPCR fragments (3.4 $\mathrm{kbp}$ from tomato and $1.8 \mathrm{kbp}$ from Japanese pear) matched the 5'untranslated region (5'-UTR) sequences of the fruit-type SS cDNAs for tomato and Japanese pear, respectively (Fig. 2). These data demonstrated that the amplified fragments contained the 5' upstream regions of the fruit-type SS genes of tomato and Japanese pear (Fig. 2). The transcription start sites of the isolated 5 ' regions of SS genes of tomato and Japanese pear were deduced by 5'-RACE analysis (Fig. 1, see also sequence data on the database: accession numbers AB455486 and AB455487). The analysis by 5'-RACE indicated that the amplified 5' region of tomato SS gene contained an intron approximately $1.6 \mathrm{kbp}$ in length within the 5'-UTR (+100 to +1687$)$, which is consistent with the report by García-Rodríguez et al. ${ }^{5}$. By comparison, the equivalent 5' region of the Japanese pear SS gene (PypSUS1) had no intron. The 5' regions of the tomato and Japanese pear SS genes upstream of the transcription start sites contained known regulatory motifs ${ }^{24}$ for phloem expression (Table 1). These two regions (approximately 3.4 $\mathrm{kbp}$ and $1.8 \mathrm{kbp}$, respectively) were fused to the GUS gene ${ }^{6}$ and then each chimeric gene was used to transform tomato. Transgenic tomatoes were grown in a contained greenhouse and two plants transformed with pTSSG-7 (TOMSSF 5':: GUS, Fig. 1) and four plants transformed with pPSSG-8 (PypSUS1 5'::GUS, Fig. 1) were selected on the basis of GUS staining strength. The presence of the transgenes in the selected transgenic tomato plants was confirmed by PCR analysis (data not shown). These plants were used for further study.

Histochemical analysis of GUS activity in tomato plants transformed with pTSSG-7 or pPSSG-8 showed a high level of GUS activity in fruit and comparably weaker activity in flower tissues (Table 2). Analysis of the staining pattern in the fruit of all transformants showed staining specific to vascular tissues and the surface of developing seeds (Fig. 3). The strongly stained region in mature seeds was testa (Table 2, Fig. 3). A high level of GUS activity was 
A

pPSP511-5 insert 3'

PypSUS1 mRNA 5' cttgtcgctcctcatccaacacttgcagaacacagacgaag

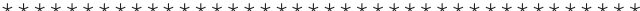

cttgtcgctcctcatccaacacttgcagaacacagacgaagaagaa (gac) 5 (gaa) ${ }_{2}$ agatagttgaatcATGg---

B

PTSPM1 insert 3'

TOMSSF MRNA 5'

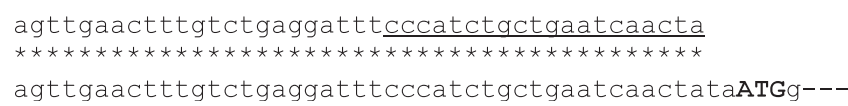

agttgaactttgtctgaggatttcccatctgctgaatcaactataATGg---

Fig. 2. Alignment of 3' sequences of IPCR fragments and 5'-UTR sequences of SS mRNAs from tomato and Japanese pear A: The pPSP511-5 insert is an IPCR product from Japanese pear. Asterisks indicate matched bases between the 3' sequence of IPCR product and the 5' sequence of Japanese pear SS (PypSUS1) mRNA. The underlined sequence shows the position of one primer (PypSUS54-34) used for IPCR. The translation initiation codon within the 5' sequence of PypSUS1 mRNA is shown in upper case font. B: The pTSPM1 insert is an IPCR product from tomato. Asterisks indicate matched bases between the 3' sequence of the IPCR product and the 5' sequence of tomato SS (TOMSSF) mRNA. The underlined sequence shows the position of one primer (LeSUS60-41) used for IPCR. The translation initiation codon within the 5' sequence of TOMSSF mRNA is shown in uppercase font.

Table 1. Sequences in SS promoters similar to phloem-specific promoter regulatory elements

\begin{tabular}{llll}
\hline \hline Element type & Consensus sequence* & $\begin{array}{l}\text { TOMSSF 5' } \\
\text { location of element** }\end{array}$ & $\begin{array}{l}\text { PypSUS1 5' } \\
\text { location of element** }\end{array}$ \\
\hline CCCC sequence & $\underline{\mathrm{CCCC}}$ & $\mathrm{NI}$ & $-183,-452,-457,-462,-676$ \\
ASL box & $\mathrm{GCA}(\mathrm{N})_{8-15}$ GCA & $\mathrm{NI}$ & $-201,-1073$ \\
GATA motif & $\mathrm{A}(\mathrm{N})_{3} \underline{\mathrm{G} A T A}$ & $-157,-450,-1154$ & -787 \\
\hline
\end{tabular}

*: Sources of those sequences are rice tungro bacilliform virus promoter ${ }^{24}$.

$* *$ : Location of underlined base of consensus sequence from transcription start site.

NI: not identified.

also observed in the outer pericarp of young (20-45 DAA) fruit of tomato plants transformed with pTSSG-7 but not with pPSSG-8 (Fig. 3).

The highest level of GUS activity was found in fruit at the early and middle stages of development in all lines transformed with pTSSG-7 or pPSSG-8 (Fig. 4). The fruit of plants transformed with pTSSG-7 had a higher level of GUS activity compared with fruit of plants transformed with pPSSG-8 (Fig. 4). Northern blot analysis of the level of GUS mRNA in transformed fruits showed a similar trend to that of GUS activity in transformed fruits (Fig. 5). However, these trends differed slightly for GUS activity and the GUS mRNA level in fruit of the 25-3 line transformed with pPSSG-8 (Figs. $4 \& 5$ ). This difference may be due to differences in the maturity of the fruits used for Northern and enzymatic analyses.

\section{Discussion}

We isolated the 5' upstream promoter region of the fruit-type SS gene of tomato and Japanese pear by IPCR (Figs. 1 \& 2). Expression characteristics of these promoters were analyzed in tomato carrying the promoter-GUS fusion gene (pTSSG-7 or pPSSG-8, Fig. 1). Histochemical analysis showed high GUS activity in the fruit of all transformants generated with pTSSG-7 and pPSSG-8 with activity specific to vascular tissues and testae (Table 2, Fig. 3). The presence of known regulatory motifs responsible for the phloemspecific expression in the 5' upstream regions supports our results of vascular expression of the SS promoters (Table 1). Furthermore, the results of vascular expression of the TOMSSF 5' (pTSSG-7 construct) are consistent with the results of in situ mRNA analysis ${ }^{21}$. These data indicate that both 5' regions have vascular type promoter activity in tomato. On the other hand, testae and vascular (phloem) tissues correspond to the transport pathways of assimilates. Although the exact localizations of TOMSSF and PypSUS1 enzyme in the vascular tissues are necessary to be clarified, it implies that TOMSSF and PypSUS1 enzymes localized in testae and vascular tissues (Table 2, Fig. 3) participate in the 


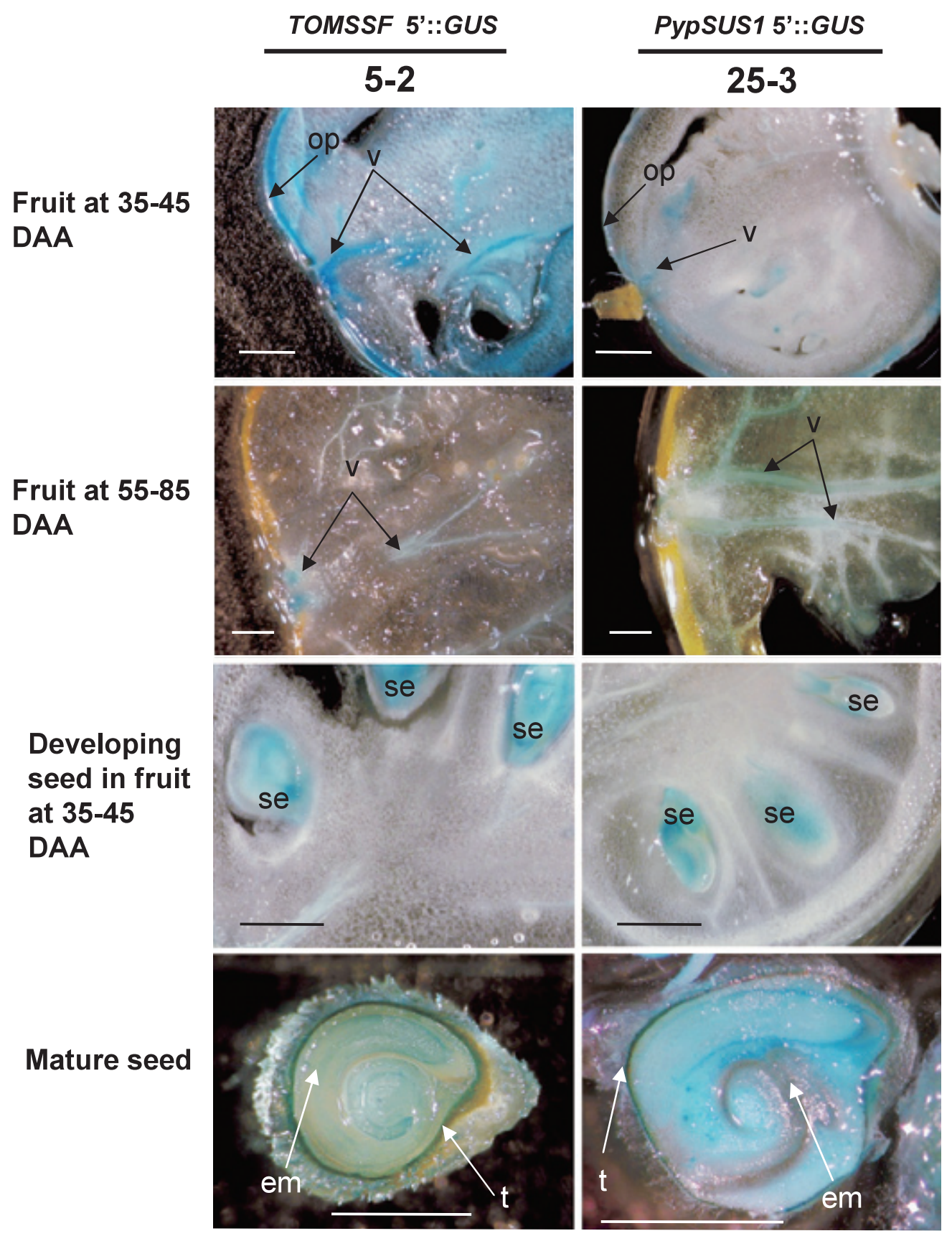

Fig. 3. Histochemical analysis of GUS activity in tomato plants (cv. Micro-Tom) carrying the TOMSSF 5'::GUS (line 5-2 transformed with pTSSG-7) or the PypSUS1 5'::GUS (line 25-3 transformed with pPSSG-8)

Transgenic tomatoes were grown in a greenhouse and then fruit at the mature green stage (35-45 DAA) or red stage (55-85 DAA) were sampled for GUS staining ( $b a r=2 \mathrm{~mm}$ ). Em, embryo; op, outer pericarp; se, seeds; t, testa; v, vascular tissue.

sucrose unloading for development of fruit and seeds. The report of D'Aoust et al. ${ }^{2}$ that antisense inhibition of TOMSSF led to decrease in sucrose import capacity in the very young fruit may support this hypothesis.

Overall expression patterns of TOMSSF 5, were similar to those of PypSUS1 5' (Table 2, Fig. 3). However, a difference in the GUS expression between TOMSSF 5, and PypSUS1 5'was observed in the outer pericarp (Fig. 3). It implies that the expression of TOMSSF leads to faster sucrose utilization in the growing pericarps of young tomato fruit as suggested by Obiadalla-Ali et al. ${ }^{9}$.

The level of GUS activity in young (20-45 DAA) fruits transformed with pTSSG-7 was higher than that in fruits transformed with pPSSG-8 (Fig. 4). The relatively 
Table. 2. Relative strength of GUS histochemical staining

\begin{tabular}{|c|c|c|c|c|}
\hline \multirow[b]{2}{*}{ Tissue } & \multicolumn{2}{|c|}{ TOMSSF 5 '::GUS } & \multicolumn{2}{|c|}{ PypSUS1 5'::GUS } \\
\hline & Line 5-2 & Line $16-10$ & Line $25-3$ & Line $29-29$ \\
\hline Leaf & - & - & - & - \\
\hline Stem & + & \pm & \pm & $\mathrm{NE}$ \\
\hline Ovary & + & ++ & + & - \\
\hline Style & - & $\mathrm{NE}$ & + & - \\
\hline Stigma & - & $\mathrm{NE}$ & + & - \\
\hline Anther & + & $\mathrm{NE}$ & + & + \\
\hline Petal & - & $\mathrm{NE}$ & - & - \\
\hline Sepal & - & $\mathrm{NE}$ & + & - \\
\hline Fruit (20-30DAA) & ++ & - & \pm & - \\
\hline Fruit (35-45DAA) & +++ & ++ & + & ++ \\
\hline Fruit (55-85DAA) & + & ++ & ++ & ++ \\
\hline Mature seed (testa) & +++ & +++ & +++ & +++ \\
\hline Root & - & - & - & - \\
\hline
\end{tabular}

NE: not examined; +++: strong activity detected; -: no activity detected; DAA: days after anthesis.

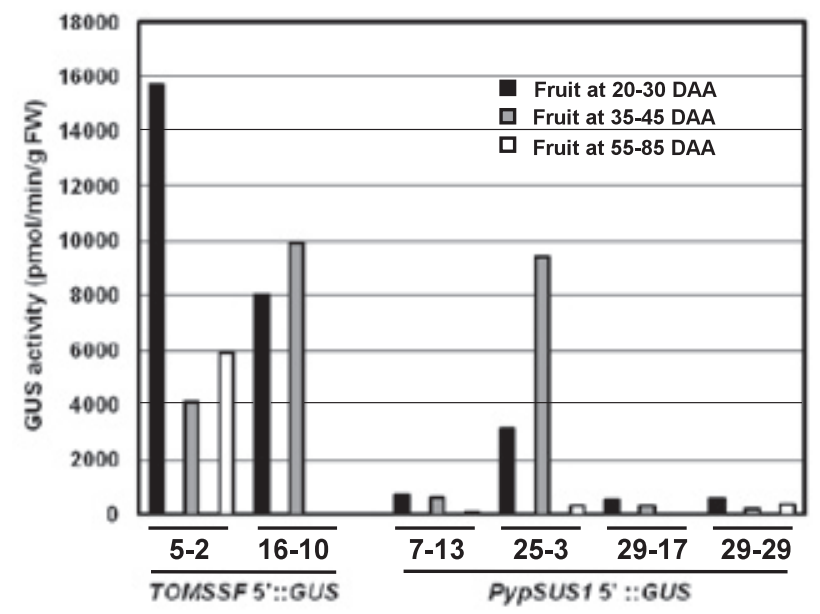

Fig. 4. GUS activity in fruit of tomato lines transformed with pTSSG-7 (TOMSSF 5'::GUS) or pPSSG-8 (PypSUS1 5'::GUS)

Fruit at various developmental stages (20-30 DAA, 35-45 DAA, and 55-85 DAA) were sampled and then the GUS activity in each fruit was measured.

high level expression of GUS in pTSSG-7 (TOMSSF 5':: $G U S$ ) fruits may be due to the presence of the intron ${ }^{10}$ in the 5'-UTR of TOMSSF (Fig. 1). The highest level of GUS activity and GUS mRNA was found in fruit at the early and middle stages of development in all transformed lines (Figs. $4 \& 5$ ). A few reports describe promoters that can drive the expression of foreign genes in young fruit of tomato. The promoters isolated in the present study can be considered as candidate tools for the genetic engineering of tomato plants

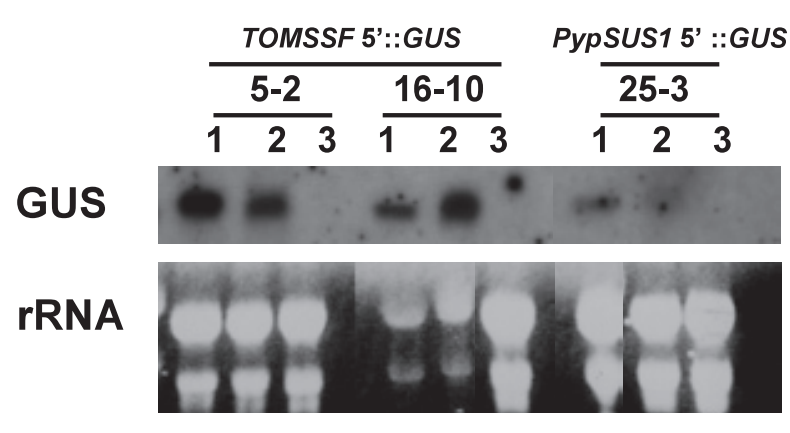

Fig. 5. Northern blot analysis of tomato fruits transformed with pTSSG-7 (TOMSSF 5'::GUS) or pPSSG-8 (PypSUS1 5'::GUS)

Total RNAs were extracted from fruit at various developmental stages (1: 20-30 DAA, 2: 35-45 DAA, 3: 55-85 DAA), electrophoresed under denaturing conditions, blotted onto a membrane, and then the GUS mRNA was detected as described in Materials and methods. The rRNA bands on the same gel were visualized by ethidium bromide staining as a loading control.

to achieve vascular expression of foreign genes at earlier stages of fruit development.

The level of GUS expression driven by the TOMSSF 5, was high at the young to middle stages of fruit development (Figs. $4 \& 5$ ). Our results for the expression patterns of GUS are consistent with the endogenous expression of TOMSSF mRNA in tomato reported by Wang et al. ${ }^{21}$. The level of PypSUS1 protein in Japanese pear fruit is reported to be high at the young stage of development then to decline 
with fruit enlargement and increases again with maturation of the fruit ${ }^{19}$. Although both TOMSSF 5' and PypSUS1 5' conferred dominant promoter activities on tomato, we did not observe a rise in the level of GUS activity or GUS mRNA in the ripening tomato fruit of plants carrying PypSUS1 5':: GUS (Figs. 4 \& 5). The production of PypSUS1 in ripening Japanese pear fruit is thought to be associated with sucrose synthesis $^{19}$. By comparison, a rise in SS activity and associated sucrose synthesis are not observed in ripe tomato fruit $^{13,16,23}$. Therefore, it is possible that the absence of a rise in GUS expression in ripening tomato fruit transformed with pPSSG-8 (PypSUS1 5'::GUS) is due to differences in genetic background between Japanese pear and tomato that relate to the mechanisms contributing to sucrose synthesis in ripening fruit.

\section{Acknowledgments}

We thank H. Maeda, K. Tanaka, and S. Negoro for their technical assistance. This work was supported by a grant from the Ministry of Agriculture, Forestry and Fisheries of Japan (Recombinant Plant Project, No. 3110).

\section{References}

1. Arai, M., Mori, H. \& Imaseki, H. (1992) Cloning and sequence of cDNAs of intracellular invertase from etiolated hypocotyl of mung bean and expression of the gene during growth of seedlings. Plant Cell Physiol., 33, 245-252.

2. D'Aoust, M. A., Yelle, S. \& Nguyen-Quoc, B. (1999) Antisense inhibition of tomato fruit sucrose synthase decreases fruit setting and the sucrose unloading capacity of young fruit. Plant Cell, 11, 2407-2418.

3. Does, M. P. et al. (1991) A quick method to estimate the TDNA copy number in transgenic plants at an early stage after transformation, using inverse PCR. Plant Mol. Biol., 17, 151-153.

4. Fu, H. \& Park, W. D. (1995) Sink- and vascular-associated sucrose synthase functions are encoded by different gene classes in potato. Plant Cell, 7, 1369-1385.

5. García-Rodríguez, S., Azcón-Aguilar, C. \& Ferrol, N. (2007) Transcriptional regulation of host enzymes involved in the cleavage of sucrose during arbuscular mycorrhizal symbiosis. Physiol. Plant., 129, 737-746.

6. Jefferson, R. A., Kavanagh, T. A. \& Bevan, M. W. (1987) GUS fusions: $\beta$-glucuronidase as a sensitive and versatile gene fusion marker in higher plants. EMBO J., 6, 39013907.

7. McCarty, D. R., Shaw, J. R. \& Hannah, L. C. (1986) The cloning, genetic mapping, and expression of the constitutive sucrose synthase locus of maize. Proc. Natl. Acad. Sci. USA, 83, 9099-9103.

8. Murray, M. G. \& Thompson, W. F. (1980) Rapid isolation of high molecular weight plant DNA. Nucleic Acids Res., 8, 4321-4325.

9. Obiadalla-Ali, H. et al. (2004) Developmental analysis of carbohydrate metabolism in tomato (Lycopersicon esculentum cv. Micro-Tom) fruits. Physiol. Plant., 120, 196-204.

10. Ohta, S. et al. (1990) Construction and expression in tobacco of a $\beta$-glucuronidase (GUS) reporter gene containing an intron within the coding sequence. Plant Cell Physiol., 31, 805-813.

11. Ohyama, A., Nishimura, S. \& Hirai, M. (1998) Cloning of cDNA for a cell wall-bound acid invertase from tomato (Lycopersicon esculentum) and expression of soluble and cell wall-bound invertases in plants and wounded leaves of L. esculentum and L. peruvianum. Genes Genet. Syst., 73, 149-157.

12. Ohyama, A. et al. (2006) Characterization of the promoter of the Wiv-1 (Lin6) gene encoding a wound-inducible cell wall-bound acid invertase in tomato. Plant Biotechnol., 23, 365-371.

13. Robinson, N. L., Hewitt, J. D. \& Bennett, A. B. (1988) Sink metabolism in tomato fruit. I. Developmental changes in carbohydrate metabolizing enzymes. Plant Physiol., 87, 727730 .

14. Salanoubat, M. \& Belliard, G. (1987) Molecular cloning and sequencing of sucrose synthase cDNA from potato (Solanum tuberosum L.): preliminary characterization of sucrose synthase mRNA distribution. Gene, 60, 47-56.

15. Sambrook, J. \& Russell, D. W. (2001) Molecular cloning: a laboratory manual. 3rd ed. Cold Spring Harbor Laboratory Press, Cold Spring Harbor, New York.

16. Schaffer, A. A. \& Petreikov, M. (1997) Sucrose-to-starch metabolism in tomato fruit undergoing transient starch accumulation. Plant Physiol., 113, 739-746.

17. Šebková, V. et al. (1995) Biochemical, physiological, and molecular characterization of sucrose synthase from Daucus carota. Plant Physiol., 108, 75-83.

18. Tanase, K. \& Yamaki, S. (2000) Sucrose synthase isozymes related to sucrose accumulation during fruit development of Japanese pear (Pyrus pyrifolia Nakai). J. Japan. Soc. Hort. Sci., 69, 671-676.

19. Tanase, K. et al. (2002) Change in the phosphorylation state of sucrose synthase during development of Japanese pear fruit. Physiol. Plant., 114, 21-26.

20. Wang, F. et al. (1993) Sucrose synthase, starch accumulation, and tomato fruit sink strength. Plant Physiol., 101, 321-327.

21. Wang, F., Smith, A. G. \& Brenner, M. L. (1994) Temporal and spatial expression pattern of sucrose synthase during tomato fruit development. Plant Physiol., 104, 535-540.

22. Werr, W. et al. (1985) Structure of the sucrose synthase gene on chromosome 9 of Zea mays L. EMBO J., 4, 1373-1380.

23. Yelle, S. et al. (1988) Sink metabolism in tomato fruit. III. Analysis of carbohydrate assimilation in a wild species. Plant Physiol., 87, 737-740.

24. Yin, Y., Chen, L. \& Beachy, R. (1997) Promoter elements required for phloem-specific gene expression from RTBV promoter in rice. Plant J., 12, 1179-1188. 